\title{
Three-dimensional Contact of a Rigid Roller Traversing a Viscoelastic Half Space
}

\author{
Carl Panek \\ Ebasco Services, Incorporated, 2 World Trade Center, \\ 89th Floor, New York, New York 10048, U.S.A. \\ AND \\ J. J. KALKER \\ Department of Mathematics, \\ Delft University of Technology, Delft, The Netherlands
}

[Received 1 July 1980]

\begin{abstract}
In this paper, the authors consider a nontrivial three-dimensional viscoelastic contact problem which has some physical significance. (Although the subject of the analysis is an elliptical roller, it is only a small step onward to the consideration of a crowned cylindrical roller.)

Generally, it is the intractability of the mathematics which hinders analytic solution of true three-dimensional problems in (visco)elasticity. The traditional method of surmounting this difficulty is to reduce the problem to two dimensions, either by choosing a suitable geometry, or by using an appropriate co-ordinate system. The elastic line integral theory represents another approach; certain approximations are used to simplify the governing equations, thus allowing the solution of the problem.

After the development of a viscoelastic analogue of the Boussinesq equation valid for the solution of quasi-steady state viscoelastic contact problems, analysis proceeds making use of near field and extended line integral approximations. Results are generated showing the velocity dependence of several physical parameters, including the size and shape of the contact zone. One additional point of interest is uncovered, namely the presence of a pressure peak near the leading edge of the contact zone.
\end{abstract}

\section{Introduction}

IN 1961, Hunter presented the solution to the two-dimensional problem of a rigid cylinder rolling on a viscoelastic half space (Hunter, 1961). For reasons of simplifying the solution to the problem, the viscoelastic solid was idealized as being a standard linear material with a single relaxation time and a constant Poisson's ratio; furthermore, inertia effects were neglected. Later, Morland extended Hunter's results by considering contact between two cylinders, and by taking into account more general constitutive laws (Morland, 1962, 1967a, b).

In the present paper the authors consider the three-dimensional version of Hunter's problem, still retaining the simplification afforded by employing the standard linear viscoelastic model and neglecting inertia effects. The actual three-dimensional analysis is done in an approximate manner by employing the elastic line contact theory (Kalker, 1972, 1977; Panek \& Kalker, 1977). Pertinent line contact equations 
which were not included in the original publications are developed in the body of this paper.

To state that the analysis is restricted to frictionless contact developed by an indentor traversing a viscoelastic half space is to point out the fact that in this analysis all rolling friction is neglected. In actuality, some shear loading would develop between a roller and the surface of the half space due to non-uniform lateral and transverse surface deformations, but the inclusion of such effects are outside the scope of this paper.

\section{The Boussinesq Equation for a Viscoelastic Solid}

Generally, this paper considers the contact between an indentor and a viscoelastic half space. For reasons of simplifying the following analysis it is assumed that the viscoelastic material has a constant Poisson's ratio, and that the behaviour of the material can be described by the standard linear model with a single relaxation time (see Fig. 1). In particular, the creep-strain behaviour of the material in shear, $e(t)$, in response to a constant stress, $\sigma$, applied at time $t=0$ is

$$
e(t)=\sigma \mu_{D}^{-1}\left[1+f\left(1-e^{-t / \tau}\right)\right] H(t),
$$

where $\mu_{D}$ is the dynamic shear modulus, $\tau$ is the relaxation time, $f$ is the ratio of the creep response to the instantaneous response and $H(t)$ is Heaviside's unit step function.

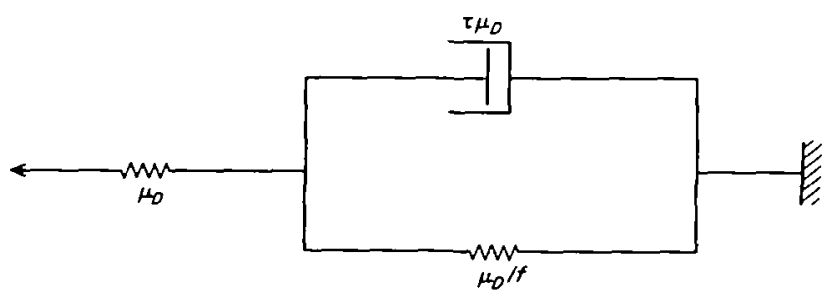

FIG. 1. Standard linear viscoelastic model.

Hunter found that for an applied load $p(x, y)$ moving across the surface of the viscoelastic solid with a constant velocity $V$, the normal surface displacement in terms of a moving co-ordinate system is given by

$$
u(x, y)=u_{e}(x, y)+f \int_{0}^{\infty} e^{-\eta} u_{e}(x+V \tau \eta, y) d \eta,
$$

where $u_{e}(x, y)$ is the instantaneous elastic displacement. This elastic displacement is given by the Boussinesq equation,

$$
u_{e}(x, y)=\frac{1-v}{2 \pi \mu_{D}} \iint_{\Lambda} \frac{p\left(x^{\prime}, y^{\prime}\right)}{\left[\left(x-x^{\prime}\right)^{2}+\left(y-y^{\prime}\right)^{2}\right]^{\frac{1}{2}}} d x^{\prime} d y^{\prime},
$$

where $v$ is Poisson's ratio and $\Lambda$ denotes the domain of the moving loaded zone. It should be noted that Equation (2) is valid for velocities $V$ small in comparison to the 
sound propagation velocities for the viscoelastic medium. To cast Equation (2) in to a form more suitable for analysis, first notice that

$$
\int_{0}^{\infty} e^{-\eta} \frac{\partial}{\partial \eta} u_{e}(x+V \tau \eta, y) d \eta=-u_{e}(x, y)+\int_{0}^{\infty} e^{-\eta} u_{e}(x+V \tau \eta, y) d \eta .
$$

By adding Equations (2) and (4) together, it is possible to eliminate the single term $u_{e}(x, y)$, and one finds

$$
u(x, y)=\int_{0}^{\infty} e^{-\eta}\left[(1+f) u_{e}(x+V \tau \eta, y)-V \tau \frac{\partial}{\partial x} u_{e}(x+V \tau \eta, y)\right] d \eta .
$$

The second term in the integral of Equation (5) comes from the fact that

$$
V \tau \frac{\partial}{\partial x} u_{e}(x+V \tau \eta, y)=\frac{\partial}{\partial \eta} u_{e}(x+V \tau \eta, y) \text {. }
$$

Defining a pseudo load according to

$$
p^{*}(x, y)=\frac{1-v}{2 \pi \mu_{D}}\left[(1+f) p(x, y)-V \tau \frac{\partial}{\partial x} p(x, y)\right],
$$

it is possible to rewrite Equation (5) as

$$
u(x, y)=\int_{0}^{\infty} e^{-\eta}\left\{\iint_{\Lambda} \frac{p^{*}\left(x^{\prime} y^{\prime}\right)}{\left[\left(x+V \tau \eta-x^{\prime}\right)^{2}+\left(y-y^{\prime}\right)^{2}\right]^{\frac{1}{2}}} d x^{\prime} d y^{\prime}\right\} d \eta .
$$

The last step in simplifying the original formula commences with differentiating both sides of Equation (8) with respect to $x$. Within the integral one may replace $\partial / \partial x$ by $1 / V \tau \partial / \partial \eta$, then integration by parts gives

$$
\frac{\partial}{\partial x} u(x, y)=\frac{-1}{V \tau} \iint_{\Lambda} \frac{p^{*}\left(x^{\prime}, y^{\prime}\right)}{\left[\left(x-x^{\prime}\right)^{2}+\left(y-y^{\prime}\right)^{2}\right]^{\frac{1}{2}}} d x^{\prime} d y^{\prime}+\frac{1}{V \tau} u(x, y) .
$$

Defining a pseudo normal surface displacement by

$$
u^{*}(x, y)=u(x, y)-V \tau \frac{\partial}{\partial x} u(x, y)
$$

one finally arrives at the equation

$$
u^{*}(x, y)=\iint_{\Lambda} \frac{p^{*}\left(x^{\prime}, y^{\prime}\right)}{\left[\left(x-x^{\prime}\right)^{2}+\left(y-y^{\prime}\right)^{2}\right]^{\frac{1}{2}}} d x^{\prime} d y^{\prime} .
$$

This result may be interpreted as the viscoelastic analogue of the Boussinesq equation.

To solve the original problem of an indentor traversing a viscoelastic solid, it is sufficient to consider the viscoelastic analogue of the Boussinesq equation presented above. Given the indentor profile, one calculates a pseudo displacement field $u^{*}(x, y)$. Presuming that one knows or can somehow determine $\Lambda$, one finds a pseudo load $p^{*}(x, y)$ which satisfies Equation (11). With this result, one goes back to the differential equation defining $p^{*}(x, y)$ and solves for $p(x, y)$ using an appropriate boundary condition. 


\section{Description of the Problem}

A rigid three-dimensional indentor is allowed to slide without friction across the surface of a viscoelastic half space at constant velocity $V$. Presuming that all transient effects have died out, one may write the stress and displacement boundary conditions for the quasi-steady state response of the material in terms of a moving co-ordinate system centred on the indentor. (Refer to Figs $2 a$ and $2 b$.) Thus, one has that

and

$$
p(x, y)=0, \text { outside } \Lambda,
$$

$$
u(x, y)=S(x, y), \quad \text { inside } \Lambda,
$$

where $\Lambda$ is the projected domain of the contact zone, $p(x, y)$ the contact pressure, $u(x, y)$ the normal displacement and $S(x, y)$ defines the shape of the lower surface of the indentor.

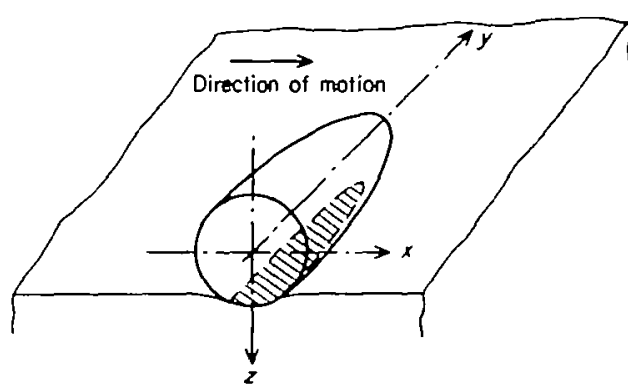

(a)

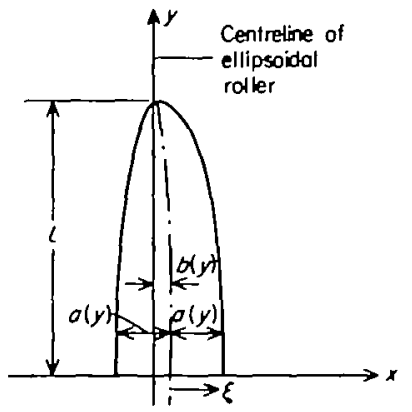

(b)

FiG. 2. Geometry of the problem.

The goal of the analysis is to determine the contact pressure, as well as the domain of contact, given the shape of the indentor. In the course of the analysis, one makes the standard assumptions of linearity and small deformations so that the boundary conditions are applied at the undeformed surface of the viscoelastic solid.

\section{The Line Integral Approximations}

Solution of the original viscoelastic problem has been reduced to the solution of an analogous elastic problem through the work of a previous section. Namely, one must satisfy Equation (11) subject to the boundary conditions given in Equations (12) and (13). Unfortunately, there is no adequate method of analytically handing the double integral on the right-hand side of Equation (11). It is for this reason that one appeals to the line contact theory. By using the line contact equations, the double integral of Equation (11) may be approximated by several single integrals plus certain functions of $x$ and $y$.

Since this particular analysis requires an extended approximation valid for all $\boldsymbol{x}$ outside the contact region in addition to the near field approximation which, as originally published (Kalker, 1972), is valid inside the contact region, it is appropriate 
to derive the extended approximation here. The following represents the simplest method for generating this approximation, and merely requires comparison of the individual near and far field approximations in the transition region. It is interesting to note that this procedure also provides a straightforward derivation of the original near field approximation.

Consider a long narrow loaded region of half length $l$ on the surface of a half space, such as would be developed by an indentor with an ellipsoidal face. For convenience, identify the $y$-direction as being along the length of the loaded zone, and the $x$ direction as being across the width of the zone (see Fig. 2b). If one further assumes that the load does not change rapidly in the $y$-direction, it is reasonable to formulate the normal surface displacements in and near the loaded region, $w_{n}$, in terms of the two-dimensional plane strain result for a loaded strip plus suitable correction terms. Accordingly, one may write

$$
\begin{aligned}
w_{n}(x, y) & =\iint_{\Lambda} \frac{p\left(x^{\prime}, y^{\prime}\right)}{\left[\left(x-x^{\prime}\right)^{2}+\left(y-y^{\prime}\right)^{2}\right]^{\frac{1}{3}}} d x^{\prime} d y^{\prime} \\
& \simeq-2 \int_{2(y)} p\left(x^{\prime}, y\right) \ln \left|x^{\prime}-x\right| d x^{\prime}+\sum_{i=0}^{\infty} A_{i}(y) x^{i},
\end{aligned}
$$

where $\lambda(y)$ is the $x$ projection of $\Lambda$ at any given $y$. [The functions $A_{l}(y)$ are yet to be determined.]

On the other hand one may compute $w_{f}$, the normal surface displacements for large $x$, by considering the action of an equivalent line load of forces and moments acting along the $y$-axis. Defining $F_{0}(y)$ and $F_{1}(y)$ according to

and

$$
F_{1}(y)=\int_{\lambda(y)} x^{\prime} p\left(x^{\prime}, y\right) d x^{\prime},
$$

one may write

$$
F_{1}(y)=\int_{\lambda(y)} x^{\prime} p\left(x^{\prime}, y\right) d x
$$

$$
\begin{aligned}
w_{f}(x, y) & =\iint_{\Lambda} \frac{p\left(x^{\prime}, y^{\prime}\right)}{\left[\left(x-x^{\prime}\right)^{2}+\left(y-y^{\prime}\right)^{2}\right]^{\frac{1}{2}}} d x^{\prime} d y^{\prime} \\
& \simeq \int_{-1}^{l} F_{0}\left(y^{\prime}\right)\left[x^{2}+\left(y-y^{\prime}\right)^{2}\right]^{-\frac{1}{2}} d y^{\prime}-\frac{\partial}{\partial x} \int_{-1}^{l} F_{1}\left(y^{\prime}\right) \times \\
& {\left[x^{2}+\left(y-y^{\prime}\right)^{2}\right]^{-1} d y^{\prime}, }
\end{aligned}
$$

where $l$ is the half length of the contact zone, and the kernel, $\left[\left(x-x^{\prime}\right)^{2}+\right.$ $\left.\left(y-y^{\prime}\right)^{2}\right]\left.^{-\frac{1}{2}}\right|_{x=0}$, is the Green's function for a line load acting along the $y$-axis $\left(x^{\prime}=0\right)$. In Equation (17), the second kernel is written in its equivalent form, as the differential of the first kernel. Doing this will facilitate some of the following work.

First, it is necessary to compare the two approximations, $w_{n}$ and $w_{f}$, in order to determine the functions $A_{i}(y)$. Once this has been done, it will be possible to develop an extended approximation which will be valid for all $x$ outside the contact region.

To allow comparison of $w_{n}$ to $w_{f}$, rewrite Equation (14) in an approximate form 
valid for $x>x^{\prime}$. Such an approximation can be made by replacing $\ln \left|x^{\prime}-x\right|$ by $\ln |x|-x^{\prime} / x-\ldots$ Doing this, one has

$$
w_{n}(x, y) \simeq-2 \int_{\lambda(y)} p\left(x^{\prime}, y\right)\left[\ln |x|-\frac{x^{\prime}}{x}-\ldots\right] d x^{\prime}+\sum_{i=0}^{\infty} A_{l}(y) x^{\prime} .
$$

Using the definitions of $F_{0}(y)$ and $F_{1}(y)$ as given in Equations (15) and (16), the above approximation becomes

$$
w_{n}(x, y) \simeq-2 F_{0}(y) \ln |x|+2 F_{1}(y) / x+\sum_{i=0}^{\infty} A_{i}(y) x^{i} .
$$

To cast Equation (17) into an approximate form valid for small $x$ (nominally $\left.x<\left|y^{\prime}-y\right|\right)$, first rearrange each integral by introducing the appropriate $F(y)$ so that typically

$$
\begin{array}{r}
\int_{-1}^{l} F\left(y^{\prime}\right)\left[x^{2}+\left(y^{\prime}-y\right)^{2}\right]^{-\frac{1}{2}} d y^{\prime}=\int_{-1}^{l}\left[F\left(y^{\prime}\right)-F(y)\right]\left[x^{2}+\left(y^{\prime}-y\right)^{2}\right]^{-1} d y^{\prime}+ \\
F(y) \int_{-1}^{l}\left[x^{2}+\left(y^{\prime}-y\right)^{2}\right]^{-\frac{1}{2}} d y^{\prime}
\end{array}
$$

Note that the second integral on the right-hand side of Equation (20) can be evaluated directly giving $\left.\sinh ^{-1}\left(\frac{y^{\prime}-y}{|x|}\right)\right|_{y^{\prime}=-1} ^{y^{\prime}=1}$. Using the logarithmic approximation for the inverse hyperbolic sine one has

$$
\left.\sinh ^{-1}\left(\frac{y^{\prime}-y}{|x|}\right)\right|_{y^{\prime}=-1} ^{y^{\prime}-1} \simeq \ln 4\left(l^{2}-y^{2}\right)-\ln \left(x^{2}\right) .
$$

For the first integral on the right-hand side of Equation (20), replace $\left[x^{2}+\left(y^{\prime}-y\right)^{2}\right]^{t}$ by $\left|y^{\prime}-y\right|$, which is consistent with the assumption that $x<\left|y^{\prime}-y\right|$. Thus, Equation (20) may be approximately reduced to

$$
\begin{aligned}
& \int_{-l}^{l} F\left(y^{\prime}\right)\left[x^{2}+\left(y^{\prime}-y\right)^{2}\right]^{-\frac{1}{2}} d y^{\prime} \simeq \int_{-l}^{l} \frac{F\left(y^{\prime}\right)-F(y)}{\left|y^{\prime}-y\right|} d y+ \\
& F(y) \ln 4\left(l^{2}-y^{2}\right)-2 F(y) \ln |x| .
\end{aligned}
$$

Using this result in Equation (17), and differentiating with respect to $x$, one finds

$$
\begin{aligned}
w_{f}(x, y) \simeq \int_{-l}^{l} \frac{F_{0}\left(y^{\prime}\right)-F_{0}(y)}{\left|y^{\prime}-y\right|} d y^{\prime}+F_{0}(y) \ln 4\left(l^{2}-y^{2}\right)- \\
2 F_{0}(y) \ln |x|+2 F_{1}(y) / x .
\end{aligned}
$$

Comparing Equation (19) with Equation (23), one concludes that for this level approximation $A_{1}(y)=A_{2}(y)=\ldots \equiv 0$, and that

$$
A_{0}(y)=\int_{-1}^{l} \frac{F_{0}\left(y^{\prime}\right)-F_{0}(y)}{\left|y^{\prime}-y\right|} d y^{\prime}+F_{0}(y) \ln 4\left(l^{2}-y^{2}\right)
$$


Consequently, one immediately arrives at the near field approximation

$$
\begin{aligned}
\iint_{\Lambda} \frac{p\left(x^{\prime}, y^{\prime}\right)}{\left[\left(x-x^{\prime}\right)^{2}+\left(y-y^{\prime}\right)^{2}\right]^{\frac{1}{2}}} d x^{\prime} d y^{\prime} \simeq & -2 \int_{\lambda(y)} p\left(x^{\prime}, y\right) \ln \left|x^{\prime}-x\right| d x^{\prime}+ \\
& F_{0}(y) \ln 4\left(l^{2}-y^{2}\right)+ \\
& \int_{-1}^{l} \frac{F_{0}\left(y^{\prime}\right)-F_{0}(y)}{\left|y^{\prime}-y\right|} d y^{\prime}, \quad|x|<\lambda(y) .
\end{aligned}
$$

Now the extended approximation, when properly formulated, should contain terms which are characteristic of both the near and far field approximations. To this end, the extended approximation will be constructed by adding the near field equation (14), to the far field equation (17), and then subtracting out the common intermediate result, Equation (19). Proceeding as indicated, one finds for the extended approximation

$$
\begin{aligned}
\iint_{\Lambda} \frac{p\left(x^{\prime}, y^{\prime}\right)}{\left[\left(x-x^{\prime}\right)^{2}+\left(y-y^{\prime}\right)^{2}\right]^{\frac{1}{2}}} d x^{\prime} d y^{\prime} & \\
= & -2 \int_{\lambda(y)} p\left(x^{\prime}, y\right) \ln \left|x^{\prime}-x\right| d x^{\prime}+ \\
& \int_{-1}^{l} F_{0}\left(y^{\prime}\right)\left[x^{2}+\left(y^{\prime}-y\right)^{2}\right]^{-\frac{1}{1}} d y^{\prime}+2 F_{0}(y) \ln |x|- \\
& \frac{\partial}{\partial x} \int_{-1}^{l} F_{1}\left(y^{\prime}\right)\left[x^{2}+\left(y^{\prime}-y\right)^{2}\right]^{-\frac{1}{1}} d y^{\prime}-2 F_{1}(y) / x, \quad|x|>\lambda(y) .
\end{aligned}
$$

\section{Solution for an Ellipsoidal Indentor}

The primary assumption necessary for the application of the line contact theory is that the width of the loaded zone be much smaller than its length. Consequently, the solution of this problem must be restricted to indentors which produce long narrow contact zones (such as bearing rollers, as opposed to narrow wheels).

Although, in the interest of reality, one refers to the indentor as an ellipsoidal roller, the first step in the solution of the problem is to invoke the concept of small deformations and approximate the lower surface of the ellipsoid by the doubly parabolic equation

$$
S(x, y)=-\frac{1}{2} C_{1} x^{2}-\frac{1}{2} C_{2} y^{2}+d,
$$

where $d$ is the depth of penetration as measured from the undeformed surface, and $C_{1}$ and $C_{2}$ are the curvatures of the ellipsoid in the lateral and longitudinal direction, respectively.

The first step is to determine, as explicitly as possible, the form of the contact pressure developed under the indentor. To this end, substitute Equation (27) into Equation (11) with its appropriate definitions, and use the near field approximation 
(25). Thus:

$$
\begin{aligned}
& \frac{1}{2} C_{1} x^{2}-V \tau C_{1} x+\frac{1}{2} C_{2} y^{2}-d \\
&=2 \int_{\lambda(y)} p^{\star}\left(x^{\prime}, y\right) \ln \left|x^{\prime}-x\right| d x^{\prime}-F_{0}(y) \ln 4\left(l^{2}-y^{2}\right)- \\
& \int_{-1}^{l} \frac{F_{0}\left(y^{\prime}\right)-F_{0}(y)}{\left|y^{\prime}-y\right|} d y^{\prime}, \text { inside } \Lambda .
\end{aligned}
$$

It is convenient to define the contact zone in terms of two new functions, $a(y)$ and $b(y)$, so that

$$
\lambda(y)=[b(y)-a(y), b(y)+a(y)], \quad|y|<l .
$$

Then, if one makes the change of variables

$$
x=\xi+b(y), \quad x^{\prime}=\xi^{\prime}+b(y), \quad|y|<l,
$$

Equation (28) may be rewritten as

$$
\begin{aligned}
2 \int_{-a(y)}^{a(y)} & p^{*}\left[\xi^{\prime}+b(y), y\right] \ln \left|\xi-\xi^{\prime}\right| d \xi^{\prime} \\
= & \frac{1}{2} C_{1} \xi^{2}-[V \tau-b(y)] C_{1} \xi+\frac{1}{2} C_{1} b^{2}(y)-V \tau C_{1} b(y)+\frac{1}{2} C_{2} y^{2}-d+ \\
& F_{0}(y) \ln 4\left(l^{2}-y^{2}\right)+\int_{-l}^{l} \frac{F_{0}\left(y^{\prime}\right)-F_{0}(y)}{\left|y^{\prime}-y\right|} d y^{\prime}, \\
& |y|<l, \quad|\xi|<a(y) .
\end{aligned}
$$

The fact that the right-hand side of Equation (31) contains terms which are either functions of $\xi$ or $y$, but not both, allows one to invert the equation. Using standard techniques (see Green \& Zerna, 1954, p. 171), one finds that

$$
\begin{aligned}
p^{*}(x, y) & =p^{*}[\xi+b(y), y] \\
& =\frac{C_{1}}{2 \pi}\left\{\sqrt{a^{2}(y)-\xi^{2}}+\frac{[V \tau-b(y)] \xi}{\sqrt{a^{2}(y)-\xi^{2}}}+\frac{G(y)}{\sqrt{a^{2}(y)-\xi^{2}}}\right\}, \quad|\xi|<a(y),
\end{aligned}
$$

where

$$
G(y)=\frac{2}{C_{1}} F_{0}(y)-\frac{1}{2} a^{2}(y), \quad|y|<l,
$$

and $F_{0}(y)$ must satisfy the integral equation

$$
\begin{aligned}
F_{0}(y) \ln \left[\frac{16\left(l^{2}-y^{2}\right)}{a^{2}(y)}\right] & +\int_{-l}^{l} \frac{F_{0}\left(y^{\prime}\right)-F_{0}(y)}{\left|y^{\prime}-y\right|} d y^{\prime} \\
& =-\frac{1}{4} C_{1} a^{2}(y)-\frac{1}{2} C_{1} b^{2}(y)+V \tau C_{1} b(y)-\frac{1}{2} C_{2} y^{2}+d, \quad|y|<l .
\end{aligned}
$$

Another equation in $G(y)$ can be determined by enforcing the condition that the contact pressure goes to zero at both the leading and trailing edges of the contact zone. This requires writing a solution to the differential equation (7) which automatically insures $p(x, y)=0$ at $x=b(y)+a(y)$, thus 


$$
\begin{aligned}
& \frac{1-v}{2 \pi \mu_{D}} p(x, y)=\frac{1}{V \tau} e^{\left(1+\Omega x / V_{\tau}\right.} \int_{x}^{b(y)+a(y)} p^{*}\left(x^{\prime}, y\right) \times \\
& e^{-\left(1+\Omega x^{\prime} / V_{t}\right.} d x^{\prime}, \quad|x-b(y)|<a(y) .
\end{aligned}
$$

Evaluating the integral in the above equation-it is advantageous to use the change of variables of Equation (30)-and imposing the condition that $p(x, y)=0$ at $x=b(y)-a(y)$, one finds that

where

$$
G(y)=\left\{[V \tau-b(y)] a(y)-\frac{a^{2}(y)}{\alpha(y)}\right\} \frac{I_{1}[\alpha(y)]}{I_{0}[\alpha(y)]}, \quad|y|<l,
$$

$$
\alpha(y)=\frac{1+f}{V \tau} a(y),
$$

and $I_{v}(x)$ is the modified Bessel function of the first kind of order $v$.

It is important to notice that Equation (11) is correctly interpreted as a relation between the shape of the bottom surface of the indentor and the contact pressure. One has not yet required that the indentor actually be in contact with the half space. Enforcing this condition will supply the fourth equation necessary to determine the four functions $F_{0}(y), G(y), a(y)$ and $b(y)$.

Now, rather than use Equation (8) to insure contact, it is simpler to find an expression for the surface displacement anywhere outside the contact zone, and then evaluate this function at the leading edge of contact. For this purpose, one may interpret (9) as a differential equation for $u(x, y)$ in terms of the double integral of $p^{*}(x, y)$. Solving this equation subject to the condition that $u(x, y) \rightarrow 0$ as $x \rightarrow \infty$, one finds

$$
u(x, y)=\frac{1}{V_{\tau}} e^{x / V \tau} \int_{x}^{\infty}\left\{\iint_{\Lambda} \frac{p^{*}\left(x^{\prime}, y^{\prime}\right)}{\left[\left(\eta-x^{\prime}\right)^{2}+\left(y-y^{\prime}\right)^{2}\right]^{\frac{1}{2}}} d x^{\prime} d y^{\prime}\right\} e^{-\eta / V \tau} d \eta,
$$

As before, the difficulty presented by the above equation is the evaluation of the double integral, and it is for this reason that the double integral is replaced by the extended approximation given in Equation (26). After making this substitution, but before integrating with respect to $\eta$, one encounters an integral involving the pseudo pressure and a logarithmic term. Since $p^{*}(x, y)$ is well defined according to Equation (32), it is possible to carry out this integration yielding

$$
\begin{aligned}
& 2 \int_{\lambda(y)} p^{*}\left(x^{\prime}, y\right) \ln \left|x^{\prime}-x\right| d x^{\prime} \\
&= \frac{1}{2} C_{1} \xi^{2}-C_{1}[V \tau-b(y)] \xi+F_{0}(y) \ln \left[\frac{a^{2}(y)}{4}\right]- \\
& \frac{1}{4} C_{1} a^{2}(y)-\frac{1}{2} C_{1} \xi \sqrt{\xi^{2}-a^{2}(y)}+C_{1}[V \tau-b(y)] \sqrt{\xi^{2}-a^{2}(y)}+ \\
& 2 F_{0}(y) \cosh ^{-1}\left|\frac{\xi}{a(y)}\right|, \quad|\xi|>a(y),
\end{aligned}
$$

where the change of variables, as well as the definition of Equation (33), has been used 
to simplify the result. With this result, and continued use of the change of variables, Equation (38) becomes

$$
\begin{aligned}
u[\xi+b(y), y]= & -\frac{1}{2} C_{1} \xi^{2}+C_{1} b(y) \xi-V \tau C_{1} b(y)+\frac{1}{4} C_{1} a^{2}(y)-F_{0}(y) \ln \left[\frac{a^{2}(y)}{4}\right]+ \\
& \frac{1}{V \tau} e^{[\xi+b(y)] / V_{\tau}} \int_{\xi+b(y)}^{\infty} e^{-\eta / V \tau}\left\{I\left[F_{0}(y), \eta\right]-\frac{\partial}{\partial \eta} I\left[F_{1}(y), \eta\right]\right\} d \eta- \\
& \frac{1}{V \tau} e^{\xi / V_{\tau}} \int_{\xi}^{\infty} e^{-\eta / V_{\tau}}\left\{-\frac{1}{2} C_{1} \eta \sqrt{\eta^{2}-a^{2}(y)}+[V \tau-b(y)] C_{1} \sqrt{\eta^{2}-a^{2}(y)}+\right. \\
& \left.2 F_{0}(y) \cosh ^{-1}\left|\frac{\eta}{a(y)}\right|\right\} d \eta, \quad|\xi|<a(y),
\end{aligned}
$$

where, typically,

$$
I[F(y), x]=\int_{-1}^{l} F\left(y^{\prime}\right)\left[x^{2}+\left(y-y^{\prime}\right)^{2}\right]^{-\frac{1}{2}} d y^{\prime}+2 F(y) \ln |x| .
$$

The second integral on the right-hand side of Equation (40) can be evaluated directly, and the second term of the first is integrated by parts. After a fair amount of substitution and manipulation, and making use of the recursion formula for modified Bessel functions of the second kind $K_{v}(x)$, one finds that at $x=b(y)+a(y)$,

$$
\begin{aligned}
F_{0}(y) \ln \left[4\left(l^{2}-y^{2}\right)\right]+ & \int_{-1}^{l} \frac{F_{0}\left(y^{\prime}\right)-F_{0}(y)}{\left|y^{\prime}-y\right|} d y^{\prime} \\
= & C_{1} e^{a(y) / V_{\mathrm{t}}}\left\{a(y) b(y) K_{1}\left[\frac{a(y)}{V \tau}\right]-G(y) K_{0}\left[\frac{a(y)}{V \tau}\right]\right\}+ \\
& \frac{1}{V \tau} e^{[b(y)+a(y)] / V_{\tau}} \int_{b(y)+a(y)}^{\infty} e^{-\eta / V_{\tau}}\left\{I\left[F_{0}(y)-\frac{1}{V \tau} F_{1}(y), \eta\right]\right\} d \eta+ \\
& \frac{1}{V \tau} I\left[F_{1}(y), b(y)+a(y)\right], \quad|y|<l .
\end{aligned}
$$

In order to evaluate the integral on the right-hand side of Equation (42), rewrite it as the difference of two integrals according to:

$$
\int_{b(y)+a(y)}^{\infty}(\cdot) d \eta=\int_{0}^{\infty}(\cdot) d \eta-\int_{0}^{b(y)+a(y)}(\cdot) d \eta .
$$

Carrying out the first integration with respect to $\eta$ yields terms containing logarithms, Bessel functions of the second kind $Y_{v}(x)$, and Struve functions $H_{v}(x)$. (See Abramowitz \& Stegun, 1965, Ch. 12.) A substitution is made in the second term before integrating. Since the term, $I\left[F_{0}(y)-F_{1}(y) / V \tau, \eta\right]$, is being integrated over the range $0<\eta<b(y)+a(y)$, it is reasonable to use the same small argument approximation which was employed when deriving Equation (22). Thus substituting, performing the integration, and rearranging terms, one has 


$$
\begin{aligned}
C_{1} e^{-b(y) / V \tau} & \left\{a(y) b(y) K_{1}\left[\frac{a(y)}{V \tau}\right]-G(y) K_{0}\left[\frac{a(y)}{V \tau}\right]\right\} \\
= & \int_{-1}^{l} \frac{Q\left(y^{\prime}\right)-Q(y)}{\left|y^{\prime}-y\right|} d y^{\prime}+Q(y) \ln \left[4\left(l^{2}-y^{2}\right)\right]+ \\
& 2 Q(y)[\gamma-\ln V \tau]-\frac{\pi}{2 V \tau} \int_{-1}^{l} Q\left(y^{\prime}\right) \times \\
& {\left[H_{0}\left(\frac{\left|y-y^{\prime}\right|}{V \tau}\right)-Y_{0}\left(\frac{\left|y-y^{\prime}\right|}{V \tau}\right)\right] d y^{\prime}, \quad|y|<l, }
\end{aligned}
$$

where $\gamma$ is Euler's constant, and

$$
Q(y)=F_{0}(y)-\frac{1}{V \tau} F_{1}(y) .
$$

The function $F_{1}(y)$ can be eliminated from Equation (45) by using its definition and evaluating the integral in $p^{*}(x, y)$. Proceeding, and making some simplification through the use of (33), one finds

$$
Q(y)=\frac{C_{1}}{2} G(y)\left[1-\frac{b(y)}{V \tau}\right] .
$$

The four equations (33), (34), (36) and (44) must be solved for the four functions $F_{0}(y), G(y), a(y)$ and $b(y)$; but in addition one must simultaneously determine the unknown half length of the contact zone $l$, which is a function of $V \tau$. This is done by requiring that the total applied load remain constant. Choosing the static indentor load as a reference value, one may write

$$
\int_{-1}^{l} F_{0}(y) d y=\left.\int_{-1}^{l} F_{0}(y) d y\right|_{V_{\tau}=0}=\left.\frac{C_{1}}{3} l a^{2}(0)\right|_{V_{\tau}=0},
$$

where the last equality of (47) is obtained by using the static result

$$
F_{0}(y)=\frac{1}{4} C_{1} a^{2}(y)=\left.\frac{1}{4} C_{1} a^{2}(0)\left(1-\frac{y^{2}}{l^{2}}\right)\right|_{V_{\tau}=0} .
$$

\section{Coefficient of Rolling Friction}

By assumption, there is no shear stress developed on the surface of the half space, and thus there is no direct frictional force. But there is an impeding force on the roller due to the non-symmetric load distribution, and from this one may calculate the coefficient of rolling friction.

Resolving the contact pressure (which acts normally to the surface of the indentor) into horizontal and vertical components, $P_{x}$ and $P_{x}$, respectively, one finds that

and

$$
P_{x}=\iint_{\Lambda} \frac{C_{1} x p(x, y)}{\left(1+C_{2}^{2} y^{2}\right)^{\frac{1}{1}}} d x d y,
$$

$$
P_{z}=\iint_{\Lambda} \frac{p(x, y)}{\left(1+C_{1}^{2} x^{2}\right)^{1}\left(1+C_{2}^{2} y^{2}\right)^{1}} d x d y
$$


Defining the coefficient of rolling friction $\chi$ as $P_{x} / P_{z}$, and using first-order approximations to the above integrals, one has

$$
\chi=C_{1} \iint_{\Lambda} x p(x, y) d x d y / \iint_{\Lambda} p(x, y) d x d y .
$$

Now it is not necessary to use Equation (35) to evaluate $\chi$. Notice that through Equation (7), one can evaluate corresponding integrals in $p^{*}(x, y)$ yielding

and

$$
\int_{\lambda(y)} p^{*}(x, y) d x=\frac{1-v}{2 \pi \mu_{D}}(1+f) \int_{\lambda(y)} p(x, y) d x,
$$

$$
\int_{\lambda(y)} x p^{*}(x, y) d x=\frac{1-v}{2 \pi \mu_{D}}\left[(1+f) \int_{\lambda(y)} x p(x, y) d x+V \tau \int_{\lambda(y)} p(x, y) d x\right] .
$$

Using these results in conjunction with Equation (51), one finds

$$
\chi=C_{1} V \tau\left\{\frac{f}{1+f}-\int_{-l}^{l} Q(y) d y / \int_{-l}^{l} F_{0}(y) d y\right\},
$$

where $F_{0}(y)$ and $Q(y)$ are as previously defined. Note that from Equation (47), the second integral of the above may be replaced with a known quantity.

\section{Numerical Solution and Results}

The most advantageous means of solving the set of simultaneous equations developed above is to define the functions $a(y)$ and $b(y)$ in terms of several unknown coefficients, and then to determine these coefficients by minimizing the residuals of the remaining equations by a least squares technique. Even so, there are several thorny numerical problems to be overcome, such as evaluating the Struve function and accurately integrating certain singular functions as required by Equation (45); but the details of such work are not within the scope of this paper.

A $5 / 1$ contact ellipse was chosen for specific analysis since this represents the broadest ellipse for which the line contact equations may be expected to give errors of less than $3 \%$ in static analysis (see Kalker, 1972, Table 1). A value of 9 was chosen for $f$ so as to accentuate the velocity dependence of the various physical quantities.

The velocity dependence of certain physical parameters describing the size and shape of the contact zone are summarized in Fig. 3. Specifically plotted are the location of the centreline of contact, and the width of the contact zone along the $x$ axis $(y=0)$, as well as the overall length of the contact zone. It is seen that as $V \tau \rightarrow \infty$, the quantities $a(0) / a_{0}\left(a_{0}\right.$ being $\left.\left.a(0)\right|_{V_{\tau}=0}\right)$ and $l / l_{0}\left(l_{0}\right.$ being $\left.l_{V_{\tau}=0}\right)$ both approach the limit $(1+f)^{-\mathfrak{t}}$ as predicted analytically.

Two other velocity dependent quantities are presented in Fig. 4. These are the coefficient of rolling friction and the depth of indentor penetration. As $V \tau \rightarrow \infty$, the quantity $d / C_{1} a_{0}^{2}$ approaches its analytic limit $\frac{1}{2}(1+f)^{-3} \ln \left(4 l_{0} / a_{0}\right)$.

Figure 5 shows resulting contact zones for increasing values of $V \tau / a(0)$. As expected, the contact zone contracts with increasing velocity, but more interesting is the pronounced shrinkage of the trailing half for intermediate velocities. Physically, 


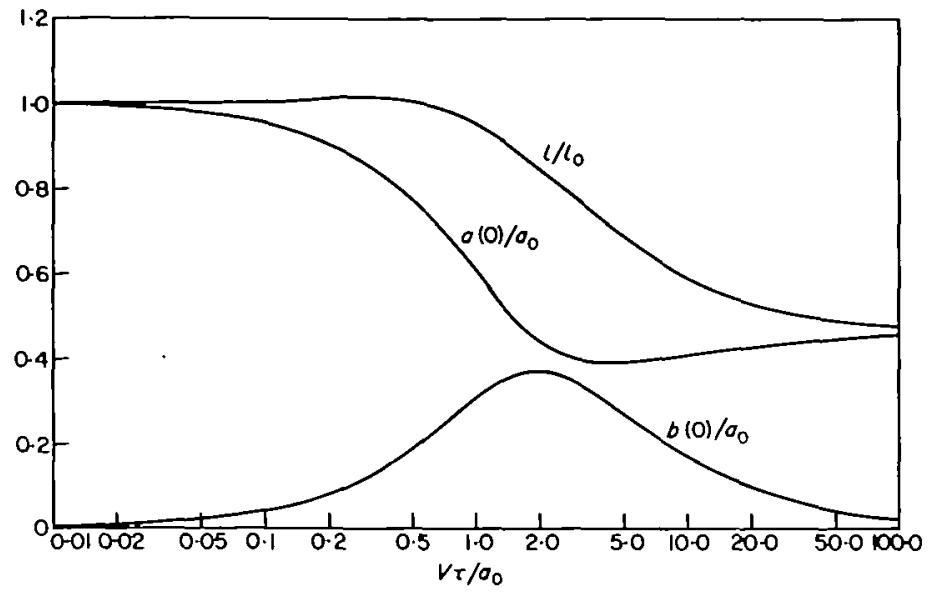

FIG. 3. Velocity dependence of certain parameters defining the size and shape of the contact zone.

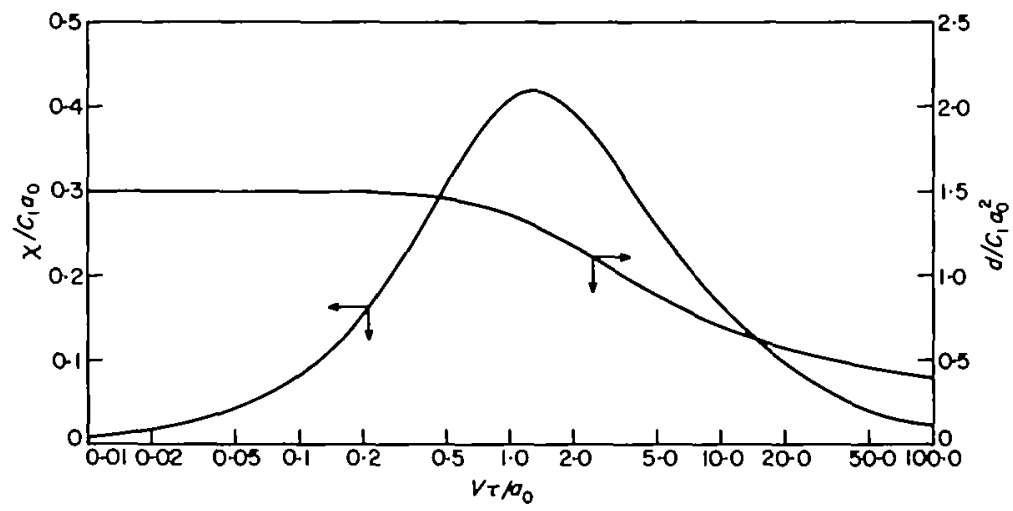

FIG. 4. Velocity dependence of rolling friction and penetration depth for the ellipsoidal roller.

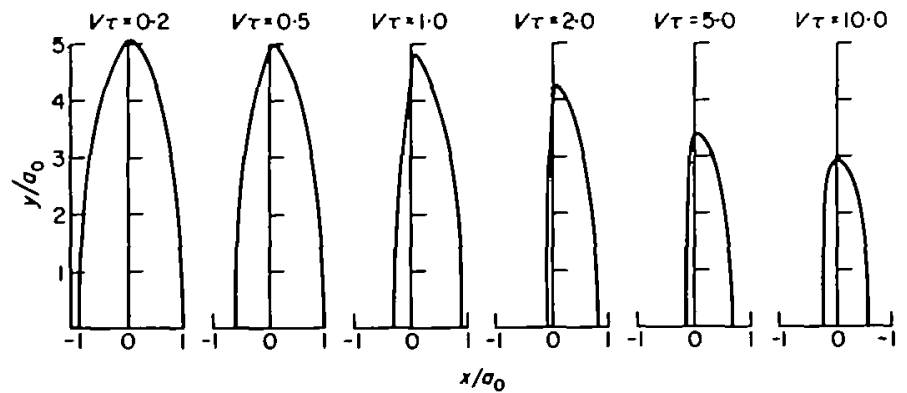

FIG. 5. Contact zone developed by the ellipsoidal roller for several different velocities. 


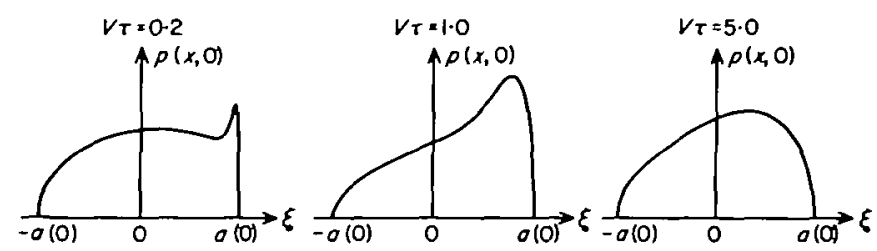

Fig. 6. Contact pressure developed along the $\xi$-axis for several different velocities (rescaled for comparison).

one should expect this because viscoelastic damping will cause a lag in recovery after indentation. One other interesting point brought out by Fig. 5 is the fact that the extreme point of the edge of the contact zone along its length (at $y=l$ ) does not lie along the indentor centreline. This is an important numerical consideration due to the pressure of the term $V \tau C_{1} b(y)$ which appears, for example, in Equation (34). Analytically, one can show that in the limit as $V \tau \rightarrow \infty$, the above term reduces to a second order function of $y$.

Figure 6 shows the contact pressure along the line $y=0$ for some values of $V \tau$. The most dramatic effect is the appearance of a pressure peak at the leading edge of contact for small velocities. This peak is verifiable analytically and is present in Hunter's solution to the two-dimensional problem, although he did not seem to notice it.

\section{Conclusions}

The elastic line contact theory, although approximate, appears to give excellent results when applied to a non-trivial three-dimensional viscoelastic contact problem. It is true that the performance criterion of the theory is merely the compliance of the analytical results to expected physical results, but this is necessary since there is no other solution to this problem in existence. The authors would be deeply indebted to anyone providing an alternative solution to this problem which would allow for more extensive evaluation of the present results. But the authors caution the users of finite element techniques to be wary of the analytically predicted pressure peak, the presence of which would require careful modelling, especially near the leading edge of contact.

The work of one of the authors (C. P.) was supported at various times by two separate fellowships granted by the Government of The Netherlands, by The University of Cincinnati, and not least of all by Ebasco Services, Incorporated.

\section{REFERENCES}

AbramowitZ, M. \& STEgUN, I. 1965 Handbook of Mathematical Functions. New York: Dover. GreEN, A. E. \& Zerna, W. 1954 Theory of Elasticity. Oxford: Clarendon Press.

HUNTER, S. C. 1961 The rolling contact of a rigid cylinder with a viscoelastic half space. $A S M E$ J. appl. Mech. 28, 611-617.

KALKER, J. J. 1972 On elastic line contact. ASME J. appl. Mech. 39, 1125-1132. 
KALKER, J. J. 1977 The surface displacement of an elastic half space loaded in a slender, bounded, curved surface region with application to the calculation of the contact pressure under a roller. J. Inst. Maths Applics 19, 127-144.

MORLAND, L. W. 1962 A plane problem of rolling contact in linear viscoelasticity theory. ASME J. appl. Mech. 29, 345-361.

MORLAND, L. W. $1967 a$ Exact solutions for rolling contact between viscoelastic cylinders. $Q . J l$ Mech. appl. Math. 20, 73-106.

MORLAND, L. W. $1976 b$ Rolling contact between dissimilar viscoelastic cylinders. $Q$. Jl appl. Math. 25, 363-376.

PANEK, C. \& KALKer, J. J. 1977 A solution for the narrow rectangular punch. J. Elasticity 2, 213-215. 